OPEN ACCESS

Edited by:

Junji Xing,

Houston Methodist Research Institute,

United States

Reviewed by:

Chunfu Zheng,

Fujian Medical University, China Rksubbarao Malireddi,

St. Jude Children's Research Hospital, United States

*Correspondence:

Xianwang Wang

275379987@qq.com

Yingying Lu

luyy39@mail.sysu.edu.cn

${ }^{\dagger}$ These authors have contributed equally to this work

Specialty section: This article was submitted to Molecular Innate Immunity,

a section of the journal

Frontiers in Immunology

Received: 15 July 2020 Accepted: 17 August 2020 Published: 02 October 2020

Citation:

Wang $X$, Yang $Y$, Cai $W-Q$ and $L U Y$ (2020) The Relationship of Sphingosine Kinase 1 With Pyroptosis Provides a New Strategy for Tumor Therapy.

Front. Immunol. 11:574990. doi: 10.3389/fimmu.2020.574990

\section{The Relationship of Sphingosine Kinase 1 With Pyroptosis Provides a New Strategy for Tumor Therapy}

\author{
Xianwang Wang ${ }^{1 *}$, Yue Yang ${ }^{1 \dagger}$, Wen-Qi Cai ${ }^{1}$ and Yingying $\mathrm{Lu}^{2 *}$ \\ ${ }^{1}$ Department of Biochemistry and Molecular Biology, Health Science Center, Yangtze University, Jingzhou, China, \\ 2 The Seventh Affiliated Hospital, Sun Yat-sen University, Shenzhen, China
}

Sphingosine kinase 1 (SPHK1) is a crucial molecule that catalyzes sphingosine to synthesize sphingosine-1-phosphate (S1P), facilitating cell survival signaling. Pyroptosis is a perplexing inflammatory mode of cell death primarily triggered by caspase-1, evoked by the NLRP3 inflammasome. Sphingosine is identified as a danger-associated molecular pattern (DAMP), which activates the NLRP3 inflammasome assembly and induces the pyroptosis. It has been demonstrated that macrophages play a pro-tumorigenic role and are closely associated with tumor progression. Attenuation of SPHK1 activity contributes significantly to macrophage pyroptosis and tumor inhibition. Calcium and integrin-binding protein 1 (CIB1) plays an important role in the translocation of SPHK1 from the cytoplasm to the plasma membrane, whereas CIB2 blocks the subcellular trafficking of SPHK1. Therefore, knockout of CIB1 or over-expression of CIB2 will result in sphingosine accumulation and contribute significantly to cancer treatment by several approaches. First, it directly provokes cancer cell apoptosis or triggers robust anti-tumor immunity by pyroptosis-induced inflammation. Second, it could restrain SPHK1 translocation from the cytoplasm to the plasma membrane and further pyroptosis, which not only drive M2 macrophages death but also facilitate tumor microenvironment inflammation as well as the further release of sphingosine from damaged macrophages. The perspective might provide novel insight into the association between SPHK1 and pyroptosis and suggest the potential target for cancer therapy.

\footnotetext{
Keywords: spingosine kinase 1, pyroptosis, calcium and integrin-binding protein 1, cancer, sphingosine1-phosphate
}

\section{INTRODUCTION}

Sphingolipids play increasingly important roles in the regulation of cell fate determination. Sphingosine kinase 1 (SPHK1) is a pivotal kinase that catalyzes ceramide and sphingosine (Sph) to yield a key sphingolipid signaling mediator sphingosine-1-phosphate (S1P), which stimulates multiple physiological processes, including cell growth, proliferation, survival, inflammation, migration, angiogenesis, etc. (1-4). Sphingosine kinase 2 (SPHK2), an isozyme of SPHK1, also has similar biological activity (Table 1). Conversely, ceramide, a second messenger in sphingolipid metabolism, triggers anti-proliferative responses involving growth inhibition, apoptosis, differentiation, and senescence $(17,18)$. Sph, generated from ceramide, also involves cell growth arrest and apoptosis (19). 
Zychlinsky and colleagues observed a lytic form of cell death in Shigella flexneri-infected macrophages referred to pyroptosis $(20,21)$. Cumulating evidence has acknowledged that pyroptosis is very perplexing, appears to be multifactorial, and is a pattern of inflammatory programmed cell death pathway activated by caspase-1, caspase-4, and caspase-5, or caspase-11. It is a pathway of cell death characterized by pore formation in the plasma membrane, cell swelling and rupture of the membrane, and massive leakage of cytosolic contents $(22,23)$. Caspase-1, a multifunctional inflammatory mediator, manages host defense against bacteria, tissue repair, tumorigenesis, metabolism, and membrane biogenesis (24). Caspase-1 is evoked by NLRP3 (Nodlike Receptor Protein 3) inflammasome, which is a multiprotein complex consisting of NLRP3 (a cytosolic mediator molecule), adaptor protein ASC (an apoptosis-associated speck-like protein containing caspase recruitment activation domain), and an effector molecule cysteine protease pro-caspase-1. These perplexing particles would be generated on exposure to cellular perturbations of intracellular microbial infections (21, 25-27). Moreover, in macrophages, priming and activation are the two essential steps for NLRP3 inflammasome activation. The priming stage is elicited by inflammatory stimuli such as TLR4 agonists, which initiates NF- $\kappa B$-induced NLRP3 and pro-IL-1 $\beta$ expression by resulting in transcriptional regulation of many inflammatory genes, involving cytokines and inflammasome components, and the activation step is driven by DAMPs (danger-associated molecular patterns) and PAMPs (pathogenrelated molecular patterns), thereby facilitating NLRP3 inflammasome formation and caspase-1-induced pyroptosis (28-31). On activation of NLRP3 inflammasome, caspase-1 mediates macrophages pyroptosis, which is controlled by the $\mathrm{N}$-terminal domain of gasdermin D (GSDMD) via assembling channels in the cell membrane and activating pro-IL-1 $\beta$ and proIL-18 for their secretion from the cells (Figure 1) (32).

A new mechanism of macrophage pyroptosis can be triggered by NETs (neutrophil extracellular trap) released by HMGB1 (high-mobility group box1) in sepsis, which can release nuclear contents of the PMN (polymorphonuclear neutrophils) into the extracellular space to trap and kill the bacteria in responding to pathogens infection (33). However, lipopolysaccharide or Gramnegative bacteria can trigger pyroptosis known as NETosis that in neutrophils, activating GSDMD cause neutrophils pyroptosis through caspase-4/11 noncanonical inflammasome signal pathway (34). Furthermore, pyroptosis has an important influence on the progress of many different neoplasms. In particular, tri-negative breast cancer, Barrett's esophageal cancer, and A549 cells can induce the occurrence of pyroptosis via caspase-1 canonical inflammasome signal pathway stimulators $(35,36)$. Especially in HCC, the expression level of NLRP3, caspase-1, IL-1 $\beta$, and IL-18 was significantly attenuated compared with that in adjacent normal tissues. Noticeably, there was a negative correlation between the pathological progress of HCC and the expression level of NLRP3 inflammasome (37-39).

Pyroptosis is a complex cell death mechanism involving multiple stimulators, pathway in which cell cytokines and inflammatory cytokines take part. Therefore, considering pyroptosis facilitation of tumor treatment requires more profound exploration. There is a balance between pro- and anti-tumorigenic roles of pyroptosis. On the one hand, pyroptosis can yield a certain microenvironment for tumorigenesis, tumor growth, and progression. On the other hand, the induction of TAMs pyroptosis can arrest tumor cell growth and development. Pyroptosis of cancer cells is also believed a potential tumor treatment approach. Thus, pro- and anti-tumorigenic roles of pyroptosis need further exploration owning to the double-edged sword of pyroptosis. In this study, the relationship of sphingosine kinase 1 on pyroptosis as it provides a new strategy for tumor therapy was discussed.

\section{SPH ACTING AS DAMP CONTRIBUTES TO THE PYROPTOSIS}

Luheshi et al. identified that both sphingosine and sphingosine analog, FTY720 can induce NLRP3-dependent activation of caspase- 1 and secretion of IL- $1 \beta$ from LPS-primed peritoneal macrophages in vitro, which was contributed to lysosomal membrane rupture, demonstrated by the translocation of cathepsin B from lysosomes to the cytosol (40). Moreover, it is revealed that cathepsin B is diffuse into the cytosol when lysosome is disrupted, accelerating a conversion in the specificity of the enzyme from an exopeptidase to an endopeptidase, efficiently cleaving SPHK1 at various sites, and leading to loss of the protein and subsequent frustration of SPHK1 activity (41). Therefore, Sph inducing lysosomal membrane rupture and resulting in the translocation of cathepsin B to the cytosol from lysosomes confers two effects. On the one hand, cathepsin B in cytoplasm cleaves SPHK1 impairing the conversion into S1P and accumulation of Sph; on the other hand, cumulative Sph acts as a DAMP, activating

TABLE 1 | The comparison between SPHK1 and SPHK2.

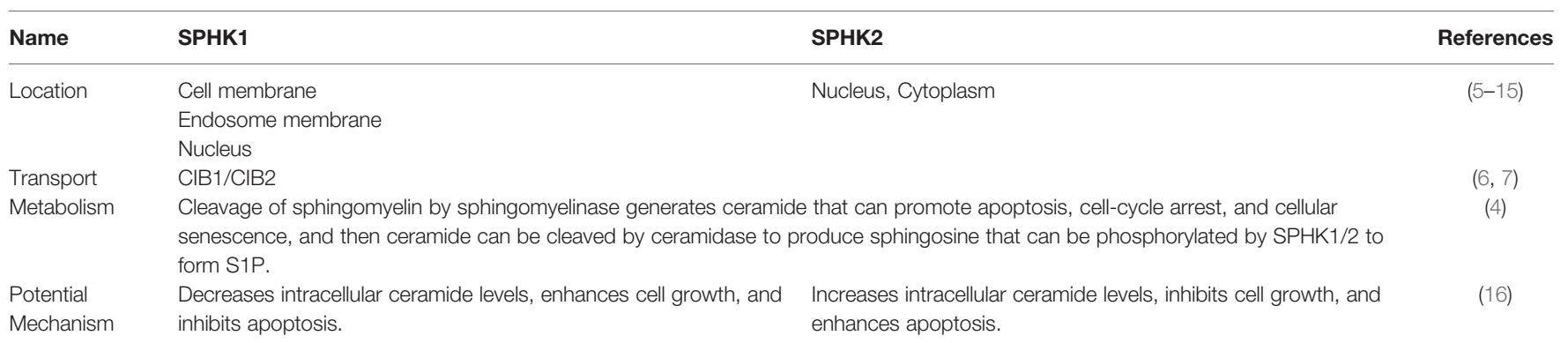




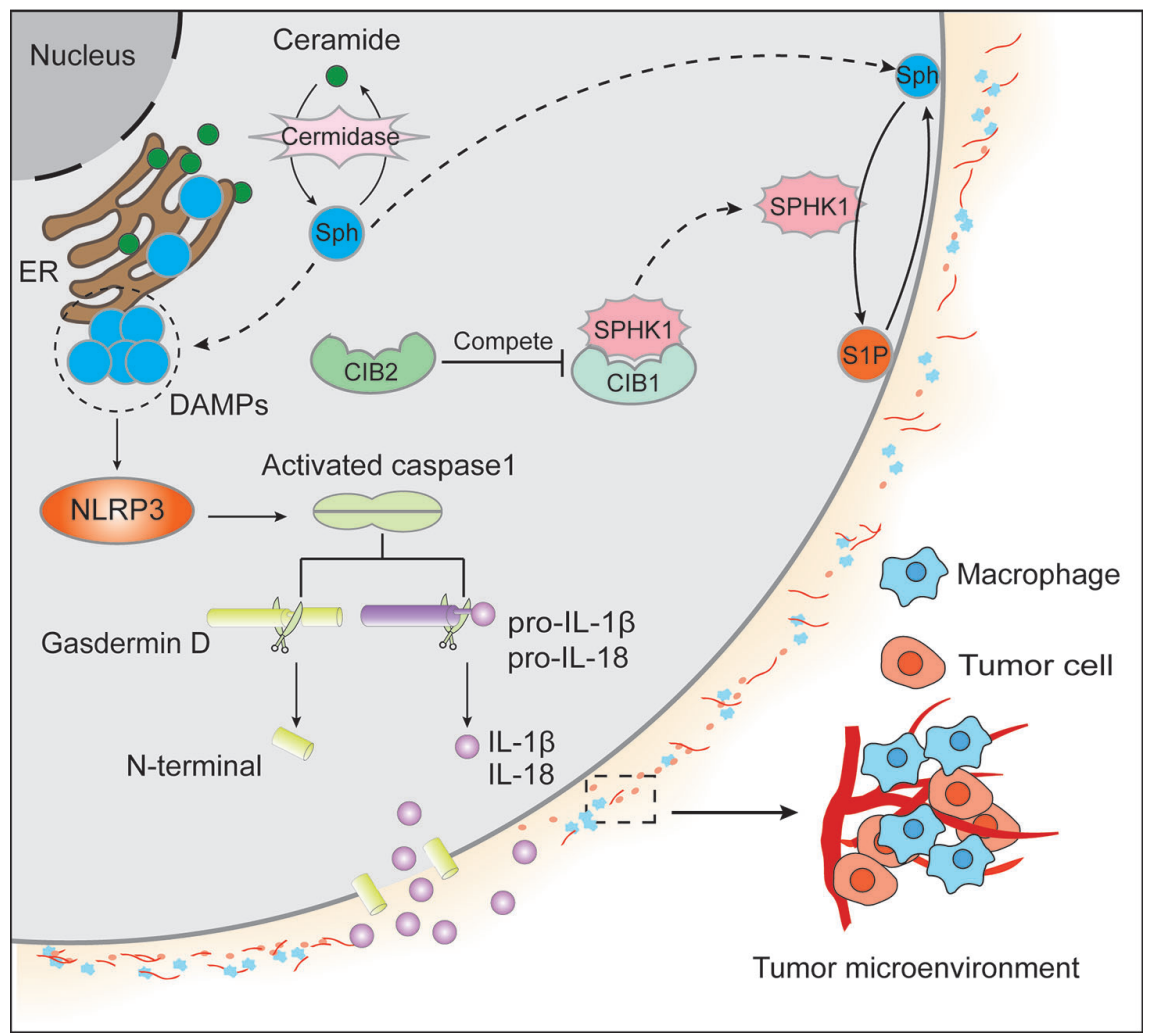

FIGURE 1 | Ceramide, primarily located in the endoplasmic reticulum membrane, generates sphingosine (Sph) by ceramidase. Both ceramide and Sph could contribute to cell apoptosis. On the one hand, Sph migrates to the cytomembrane from the endoplasmic reticulum (ER) membrane and produces sphingosine-1phosphate (S1P) by sphingosine kinase 1 (SPHK1). In contrast to the apoptotic effect of Sph, S1P could facilitate cell survival signaling. On the other hand, SPHK1 subcellular localization was regulated by calcium and integrin-binding protein 1 (CIB1) and CIB2. It is believed that the translocation of SPHK1 from the cytoplasm to the cytomembrane is dependent on CIB1, whereas CIB2 competes with CIB1 to binds to SPHK1, resulting in the cytoplasm retention of SPHK1 and the inhibition of its enzymatic activity. Additionally, the accumulation of Sph in ER acts as DAMPs to activate NLRP3 and the oligomeric RLRP3 inflammasome, further causing

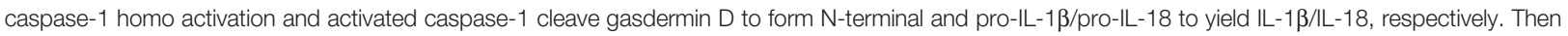
$\mathrm{N}$-terminal of gasdermin D will form the channels in the plasma membrane causing massive leakage of cytosolic contents including IL-1 $\beta$ and IL-18, which leads to tumor microenvironment inflammation in that IL-1 $\beta$ can activate primary T cells and memory T cells, IL-18 can promote interferon (IFN)- $\gamma$ production in TH1 cells, NK cells and cytotoxic T cells, boost the development of TH2 cells, and improve local inflammation response.

NLRP3 inflammasome assembly, thereby inducing pyroptosis. There are two common ways for macrophages to be activated: IL4 mediated alternative macrophage activation, which is known as alternatively activated (M2) macrophages, and classically activated (M1) macrophages activated by microbe products or PAMPs (4244). Recently researchers have characterized that tumor-associated macrophages (TAMs, M2-like functions, and phenotype) permeate into tumor tissues in large numbers, and macrophages have a protumorigenic role closely associated with tumor promotion (45). Thus, inhibiting the enzymatic activity of SPHK1 might contribute greatly to TAMs pyroptosis to promote anti-tumor progression.

\section{CIB1 MODULATES THE TRANSLOCATION OF SPHK1}

Calcium and integrin-binding protein 1 (CIB1), first discovered in 1997 as a novel interacting protein of the integrin aIIb, is a calcium modulating molecule, which is implicated in multiple cellular processes, including calcium signaling, cell growth, and proliferation, migration, adhesion, and apoptosis $(46,47)$. The myristoyl group of CIB1 is sequestered into a hydrophobic pocket in the protein's absence of intracellular $\mathrm{Ca}^{2+} \cdot \mathrm{Ca}^{2+}$ binding promotes topology changes enabling two effects as follows: first in conferring the association between protein and partners, and second to provoke the appearance of the myristoyl group from its original buried region, targeting the protein and any nascent associated interacting partner to intracellular membranes. Notably, CIB1 is essential for the agonist-induced migration of SPHK1 from the cytoplasm to the plasma membrane $(5,48)$. Jarman et al. explored how either knockdown of CIB1 or a dominant-negative CIB1 could restrain the agonist-dependent redistribution of SPHK1 (5). Zhu et al. also confirmed that overexpression of CIB1 contributes high distribution of SPHK1 to the plasma membrane, which was verified by boosted plasma membrane-associated SPHK1 activity, and attenuates the 
synthesis of S1P in CIB1 over-expressing cells (6). Although both CIB1 and CIB2 bind to SPHK1 on the $\alpha 8$ helix of SPHK1, CIB2 lacks the $\mathrm{Ca}^{2+}$-myristoyl switch function. Contrary to CIB1, CIB2 blocks the translocation of SPHK1 to the cell membrane and undermines its subsequent signaling. The interaction between CIB2 and SPHK1 is independent of $\mathrm{Ca}^{2+}$, or $\mathrm{Mg}^{2+}$, and myristoylation of CIB2 does not affect their association $(6,7)$. It is probable that CIB2 competitively binds to SPHK1 with CIB1 and gives rise to the retention of SPHK1 in the cytoplasm.

\section{SUMMARY AND PROSPECT}

Thus, a proposal that facilitated Sph release in tumor microenvironment might be an effective treatment scheme. Baroja-Mazo et al. found that NLRP3 and ASC complexes released from pyroptosis cells functioned as danger signals giving rise to amplifying inflammation by improving the activation of extracellular caspase-1 and in surrounding macrophages following internalization of the particles (16).

In summary, mutating CIB1 or over-expression of CIB2 resulting in Sph accumulation might contribute twofold to cancer therapy: in cancer cells, it directly provokes cell apoptosis, or triggers robust anti-tumor immunity by pyroptosis-induced inflammation as well as a combination with some potential antitumor drugs; in M2 macrophages, it could restrict SPHK1 redistribution from cytoplasm to cytomembrane and trigger subsequent pyroptosis, which not only causes M2 macrophages death and play an essential role in anti-tumor progression, but also facilitates both tumor microenvironment inflammation and Sph leakage from damaged macrophages. Together, our review might provide insight into the close association between SPHK1 and pyroptosis and contribute to tumor treatment's potential strategy.

\section{AUTHOR CONTRIBUTIONS}

XW and YL designed the study. XW and YY drafted the manuscript. YY and W-QC drew the figure and filled the table. XW and YL and YY revised the manuscript. All authors contributed to the article and approved the submitted version.

\section{FUNDING}

This work was supported by grants from the National Natural Science Foundation of China (31700736, 31501116), China Scholarship Council (201908420102), Hubei Medical Youth Tip-Top Talent (to XW), Leading Talent Program of Yangtze Talent Project (to XW), and the College Students Innovative Entrepreneurial Training Program in Yangtze University (2018184, 2019372).

\section{REFERENCES}

1. Johnson KR, Johnson KY, Crellin HG, Ogretmen B, Boylan AM, Harley RA, et al. Immunohistochemical distribution of sphingosine kinase 1 in normal and tumor lung tissue. J Histochem Cytochem (2005) 53:1159-66. doi: 10.1369/jhc.4A6606.2005

2. Wang Z, Min X, Xiao SH, Johnstone S, Romanow W, Meininger D, et al. Molecular basis of sphingosine kinase 1 substrate recognition and catalysis. Structure (2013) 21:798-809. doi: 10.1016/j.str.2013.02.025

3. Nava VE, Hobson JP, Murthy S, Milstien S, Spiegel S. Sphingosine kinase type 1 promotes estrogen-dependent tumorigenesis of breast cancer MCF-7 cells. Exp Cell Res (2002) 281:115-27. doi: 10.1006/excr.2002.5658

4. Maceyka M, Spiegel S. Sphingolipid metabolites in inflammatory disease. Nature (2014) 510:58-67. doi: 10.1038/nature13475

5. Jarman KE, Moretti PA, Zebol JR, Pitson SM. Translocation of sphingosine kinase 1 to the plasma membrane is mediated by calcium- and integrinbinding protein 1. J Biol Chem (2010) 285:483-92. doi: 10.1074/ jbc.M109.068395

6. Zhu W, Gliddon BL, Jarman KE, Moretti PAB, Tin T, Parise LV, et al. CIB1 contributes to oncogenic signalling by Ras via modulating the subcellular localisation of sphingosine kinase 1. Oncogene (2017) 36:2619-27. doi: 10.1038 /onc. 2016.428

7. Zhu W, Jarman KE, Lokman NA, Neubauer HA, Davies LT, Gliddon BL, et al. CIB2 Negatively Regulates Oncogenic Signaling in Ovarian Cancer via Sphingosine Kinase 1. Cancer Res (2017) 77:4823-34. doi: 10.1158/00085472.Can-17-0025

8. Inagaki Y, Li PY, Wada A, Mitsutake S, Igarashi Y. Identification of functional nuclear export sequences in human sphingosine kinase 1. Biochem Biophys Res Commun (2003) 311:168-73. doi: 10.1016/j.bbrc.2003.09.194

9. Igarashi N, Okada T, Hayashi S, Fujita T, Jahangeer S, Nakamura S. Sphingosine kinase 2 is a nuclear protein and inhibits DNA synthesis. J Biol Chem (2003) 278:46832-9. doi: 10.1074/jbc.M306577200

10. Okada T, Ding G, Sonoda H, Kajimoto T, Haga Y, Khosrowbeygi A, et al. Involvement of $\mathrm{N}$-terminal-extended form of sphingosine kinase 2 in serumdependent regulation of cell proliferation and apoptosis. J Biol Chem (2005) 280:36318-25. doi: 10.1074/jbc.M504507200

11. Ding G, Sonoda H, Yu H, Kajimoto T, Goparaju SK, Jahangeer S, et al. Protein kinase D-mediated phosphorylation and nuclear export of sphingosine kinase 2. J Biol Chem (2007) 282:27493-502. doi: 10.1074/jbc.M701641200

12. Weigert A, Cremer S, Schmidt MV, von Knethen A, Angioni C, Geisslinger G, et al. Cleavage of sphingosine kinase 2 by caspase- 1 provokes its release from apoptotic cells. Blood (2010) 115:3531-40. doi: 10.1182/blood-2009-10-243444

13. Shen H, Giordano F, Wu Y, Chan J, Zhu C, Milosevic I, et al. Coupling between endocytosis and sphingosine kinase 1 recruitment. Nat Cell Biol (2014) 16:652-62. doi: 10.1038/ncb2987

14. Dominguez G, Maddelein ML, Pucelle M, Nicaise Y, Maurage CA, Duyckaerts C, et al. Neuronal sphingosine kinase 2 subcellular localization is altered in Alzheimer's disease brain. Acta Neuropathol Commun (2018) 6:25. doi: 10.1186/s40478-018-0527-z

15. Lee JY, Han SH, Park MH, Baek B, Song IS, Choi MK, et al. Neuronal SphK1 acetylates COX2 and contributes to pathogenesis in a model of Alzheimer's Disease. Nat Commun (2018) 9:1479. doi: 10.1038/s41467-018-03674-2

16. Baroja-Mazo A, Martín-Sánchez F, Gomez AI, Martínez CM, Amores-Iniesta J, Compan V, et al. The NLRP3 inflammasome is released as a particulate danger signal that amplifies the inflammatory response. Nat Immunol (2014) 15:73848. doi: 10.1038/ni.2919

17. Ogretmen B, Hannun YA. Biologically active sphingolipids in cancer pathogenesis and treatment. Nat Rev Cancer (2004) 4:604-16. doi: 10.1038/nrc1411

18. Hannun YA. Functions of ceramide in coordinating cellular responses to stress. Science (1996) 274:1855-9. doi: 10.1126/science.274.5294.1855

19. Cuvillier O, Nava VE, Murthy SK, Edsall LC, Levade T, Milstien S, et al. Sphingosine generation, cytochrome c release, and activation of caspase- 7 in doxorubicin-induced apoptosis of MCF7 breast adenocarcinoma cells. Cell Death Differ (2001) 8:162-71. doi: 10.1038/sj.cdd.4400793 
20. Zychlinsky A, Prevost MC, Sansonetti PJ. Shigella flexneri induces apoptosis in infected macrophages. Nature (1992) 358:167-9. doi: 10.1038/358167a0

21. Jorgensen I, Miao EA. Pyroptotic cell death defends against intracellular pathogens. Immunol Rev (2015) 265:130-42. doi: 10.1111/imr.12287

22. Fink SL, Cookson BT. Caspase-1-dependent pore formation during pyroptosis leads to osmotic lysis of infected host macrophages. Cell Microbiol (2006) 8:1812-25. doi: 10.1111/j.1462-5822.2006.00751.x

23. Man SM, Karki R, Kanneganti TD. Molecular mechanisms and functions of pyroptosis, inflammatory caspases and inflammasomes in infectious diseases. Immunol Rev (2017) 277:61-75. doi: 10.1111/imr.12534

24. Man SM, Karki R, Briard B, Burton A, Gingras S, Pelletier S, et al. Differential roles of caspase-1 and caspase-11 in infection and inflammation. Sci Rep (2017) 7:45126. doi: 10.1038/srep45126

25. Siegel RM. Caspases at the crossroads of immune-cell life and death. Nat Rev Immunol (2006) 6:308-17. doi: 10.1038/nri1809

26. Martinon F, Burns K, Tschopp J. The inflammasome: a molecular platform triggering activation of inflammatory caspases and processing of prolL-beta. Mol Cell (2002) 10:417-26. doi: 10.1016/s1097-2765(02)00599-3

27. Agostini L, Martinon F, Burns K, McDermott MF, Hawkins PN, Tschopp J. NALP3 forms an IL-1beta-processing inflammasome with increased activity in Muckle-Wells autoinflammatory disorder. Immunity (2004) 20:319-25. doi: 10.1016/s1074-7613(04)00046-9

28. Yang $Y$, Wang $H$, Kouadir M, Song $H$, Shi F. Recent advances in the mechanisms of NLRP3 inflammasome activation and its inhibitors. Cell Death Dis (2019) 10:128. doi: 10.1038/s41419-019-1413-8

29. de Carvalho RVH, Silva ALN, Santos LL, Andrade WA, de Sá KSG, Zamboni DS. Macrophage priming is dispensable for NLRP3 inflammasome activation and restriction of Leishmania amazonensis replication. J Leukoc Biol (2019) 106:631-40. doi: 10.1002/jlb.Ma1118-471r

30. Krześniak M, Zajkowicz A, Gdowicz-Kłosok A, Głowala-Kosińska M, ŁasutSzyszka B, Rusin M. Synergistic activation of p53 by actinomycin D and nutlin$3 \mathrm{a}$ is associated with the upregulation of crucial regulators and effectors of innate immunity. Cell Signal (2020) 69:109552. doi: 10.1016/j.cellsig.2020.109552

31. Teng JF, Mei QB, Zhou XG, Tang Y, Xiong R, Qiu WQ, et al. Polyphyllin VI Induces Caspase-1-Mediated Pyroptosis via the Induction of ROS/NF- $\mathrm{kB} /$ NLRP3/GSDMD Signal Axis in Non-Small Cell Lung Cancer. Cancers (Basel) (2020) 12(1):193. doi: 10.3390/cancers 12010193

32. Shi J, Zhao Y, Wang K, Shi X, Wang Y, Huang H, et al. Cleavage of GSDMD by inflammatory caspases determines pyroptotic cell death. Nature (2015) 526:660-5. doi: 10.1038/nature15514

33. Chen L, Zhao Y, Lai D, Zhang P, Yang Y, Li Y, et al. Neutrophil extracellular traps promote macrophage pyroptosis in sepsis. Cell Death Dis (2018) 9:597. doi: 10.1038/s41419-018-0538-5

34. Chen KW, Monteleone M, Boucher D, Sollberger G, Ramnath D, Condon $\mathrm{ND}$, et al. Noncanonical inflammasome signaling elicits gasdermin Ddependent neutrophil extracellular traps. Sci Immunol (2018) 3(26): eaar6676. doi: 10.1126/sciimmunol.aar6676

35. Andl CD. Putting the Pieces Together: NOD-Like Receptor Protein 3 Inflammasome Priming and Activation in Barrett's Epithelial Cells. Cell Mol Gastroenterol Hepatol (2016) 2:400-2. doi: 10.1016/j.jcmgh.2016.04.004
36. Pizato N, Luzete BC, Kiffer L, Corrêa LH, de Oliveira Santos I, Assumpção JAF, et al. Omega-3 docosahexaenoic acid induces pyroptosis cell death in triple-negative breast cancer cells. Sci Rep (2018) 8:1952. doi: 10.1038/s41598018-20422-0

37. Wei Q, Mu K, Li T, Zhang Y, Yang Z, Jia X, et al. Deregulation of the NLRP3 inflammasome in hepatic parenchymal cells during liver cancer progression. Lab Invest (2014) 94:52-62. doi: 10.1038/labinvest.2013.126

38. Chu Q, Jiang Y, Zhang W, Xu C, Du W, Tuguzbaeva G, et al. Pyroptosis is involved in the pathogenesis of human hepatocellular carcinoma. Oncotarget (2016) 7:84658-65. doi: 10.18632/oncotarget.12384

39. Xia X, Wang X, Cheng Z, Qin W, Lei L, Jiang J, et al. The role of pyroptosis in cancer: pro-cancer or pro-"host"? Cell Death Dis (2019) 10:650. doi: 10.1038/ s41419-019-1883-8

40. Luheshi NM, Giles JA, Lopez-Castejon G, Brough D. Sphingosine regulates the NLRP3-inflammasome and IL-1 $\beta$ release from macrophages. Eur J Immunol (2012) 42:716-25. doi: 10.1002/eji.201142079

41. Taha TA, El-Alwani M, Hannun YA, Obeid LM. Sphingosine kinase-1 is cleaved by cathepsin B in vitro: identification of the initial cleavage sites for the protease. FEBS Lett (2006) 580:6047-54. doi: 10.1016/ j.febslet.2006.09.070

42. Gordon S, Mantovani A. Diversity and plasticity of mononuclear phagocytes. Eur J Immunol (2011) 41:2470-2. doi: 10.1002/eji.201141988

43. Gordon S, Plüddemann A, Martinez Estrada F. Macrophage heterogeneity in tissues: phenotypic diversity and functions. Immunol Rev (2014) 262:36-55. doi: $10.1111 / \mathrm{imr} .12223$

44. Stein M, Keshav S, Harris N, Gordon S. Interleukin 4 potently enhances murine macrophage mannose receptor activity: a marker of alternative immunologic macrophage activation. J Exp Med (1992) 176:287-92. doi: 10.1084/jem.176.1.287

45. Komohara Y, Fujiwara Y, Ohnishi K, Takeya M. Tumor-associated macrophages: Potential therapeutic targets for anti-cancer therapy. Adv Drug Deliv Rev (2016) 99:180-5. doi: 10.1016/j.addr.2015.11.009

46. Naik UP, Patel PM, Parise LV. Identification of a novel calcium-binding protein that interacts with the integrin alphaIIb cytoplasmic domain. J Biol Chem (1997) 272:4651-4. doi: 10.1074/jbc.272.8.4651

47. Wang X, Peng X, Zhang X, Xu H, Lu C, Liu L, et al. The Emerging Roles of CIB1 in Cancer. Cell Physiol Biochem (2017) 43:1413-24. doi: 10.1159/000481873

48. Meyer T, York JD. Calcium-myristoyl switches turn on new lights. Nat Cell Biol (1999) 1:E93-95. doi: 10.1038/12090

Conflict of Interest: The authors declare that the research was conducted in the absence of any commercial or financial relationships that could be construed as a potential conflict of interest.

Copyright $\odot 2020$ Wang, Yang, Cai and Lu. This is an open-access article distributed under the terms of the Creative Commons Attribution License (CC BY). The use, distribution or reproduction in other forums is permitted, provided the original author(s) and the copyright owner(s) are credited and that the original publication in this journal is cited, in accordance with accepted academic practice. No use, distribution or reproduction is permitted which does not comply with these terms. 